\title{
Application of Artificial Neural Network (ANN) in prediction of crystallization induction period
}

\author{
Min Yuan, Yujie Gao \\ Tianjin Academy of Environmental Sciences, Tianjin 300191, China
}

\begin{abstract}
Keywords: Industrial crystallization; Artificial neural network; Induction period
Abstract: The induction period (INT) is a measure of the ability of a supersaturated solution to remain the metastable state, and it is a very important parameter to design and optimize the crystallization process. Due to the highly nonlinear dependence of INT on the process parameters such as supersaturation and concentration, the conventional models are difficult to give prediction with high accuracy. More-accurate approaches for predicting INT are greatly needed in the design and operation of industrial crystallization process. Artificial neural network (ANN), as a non-model based prediction method, does not require any assumed special mathematic function to fit the experimental data. ANN was utilized to estimate the experimentally determined INT. The simulation results showed that the ANN could give good prediction of the INT data.
\end{abstract}

\section{Introduction}

Induction period (INT) is the critical parameter for the optimization of crystallization process and designing of crystallizer, which was commonly served as the important tool to estimate the nucleation kinetics for a given crystallization system ${ }^{1,2}$. However, the INT is especially sensitive to the crystallization process parameters such as temperature, concentration ${ }^{3-5}$. Therefore, it is essential to establish the INT prediction model to estimate the INT under different crystallization process conditions.

Unlike the traditional models, artificial neural network (ANN) model is not limited to specific mathematical equation, and it is able to simulate almost all nonlinear systems ${ }^{6}$. Since the ANN simulation method was developed, it has played important role in the development of science and technology ${ }^{7}$. Due to the strong nonlinear relation between process parameters and INT, it is difficult to describe the dependence of INT on process parameters by general mathematical equations. Therefore, traditional models tend to bring about large errors in prediction of INT.

The present work aims to apply ANN on the prediction of INT which is largely dependent on crystallization process parameters.

\section{Prediction method}

The ANN was established by simulating the structure or functional aspects of biological neural networks. A neural network is made up of interconnected artificial neurons, the structure of which is adjusted based on external or internal information that flows through the network during the learning process ${ }^{8}$.

Among different types of ANN, some reports show the multilayer perceptron (MLP) feedforward networks as a popular model in published works, which consist of an input layer, one or more hidden layers and an output layer ${ }^{9}$. Some researches demonstrate that neural network with multi-layer is more effective than single-layer neural network to approximate nonlinear relation ${ }^{10}$. 
For this reason, multi-layer neural network was adopted to construct the network in this work, describing the INT as a function of process parameters.

Some literature indicated that the number of hidden layers and the number of processing elements of each hidden layer are important for the accuracy of the simulation results. It has been proved that the ANN model consisting of one hidden layer is able to interpret any nonlinear systems $^{11}$, and so is believed to give sufficiently good approximations for predicting INT. Therefore, multilayer ANN model with single hidden layer was adopted. As the complexity in the relationship between the input data and desired output increases, the number of the processing elements in the hidden layer should also increase. The number of the processing elements in hidden layer should be determined by comparing the prediction error of each trained ANN with different number of processing elements in hidden layer.

Fig. 1 illustrates generically the architecture of the networks used in this work, in which $X$ is the input vector and $Y$ is the output vector. The parameters $W 1, W 2, b 1$ and $b 2$ represent weighting matrix of the hidden-layer and output-layer, bias vectors of the hidden-layer and output-layer respectively. $N 1$ represents the hidden-layer parameter vectors, which can be defined as

$$
N_{1}=W 1 \times X+b 1
$$

The hidden-layer propagation function, $f_{1}(x)$, is expressed as

$$
f_{1}(x)=\frac{2}{1+e^{-2 x}}-1
$$

Its purpose is to send information in the hidden layer to the output layer. The output parameter, $N_{2}$, can be calculated using the following equation:

$$
N_{2}=W 2 \times H+b 2
$$

The definition of the output-layer propagation function, $f_{2}(x)$, is

$$
f_{2}(x)=x
$$

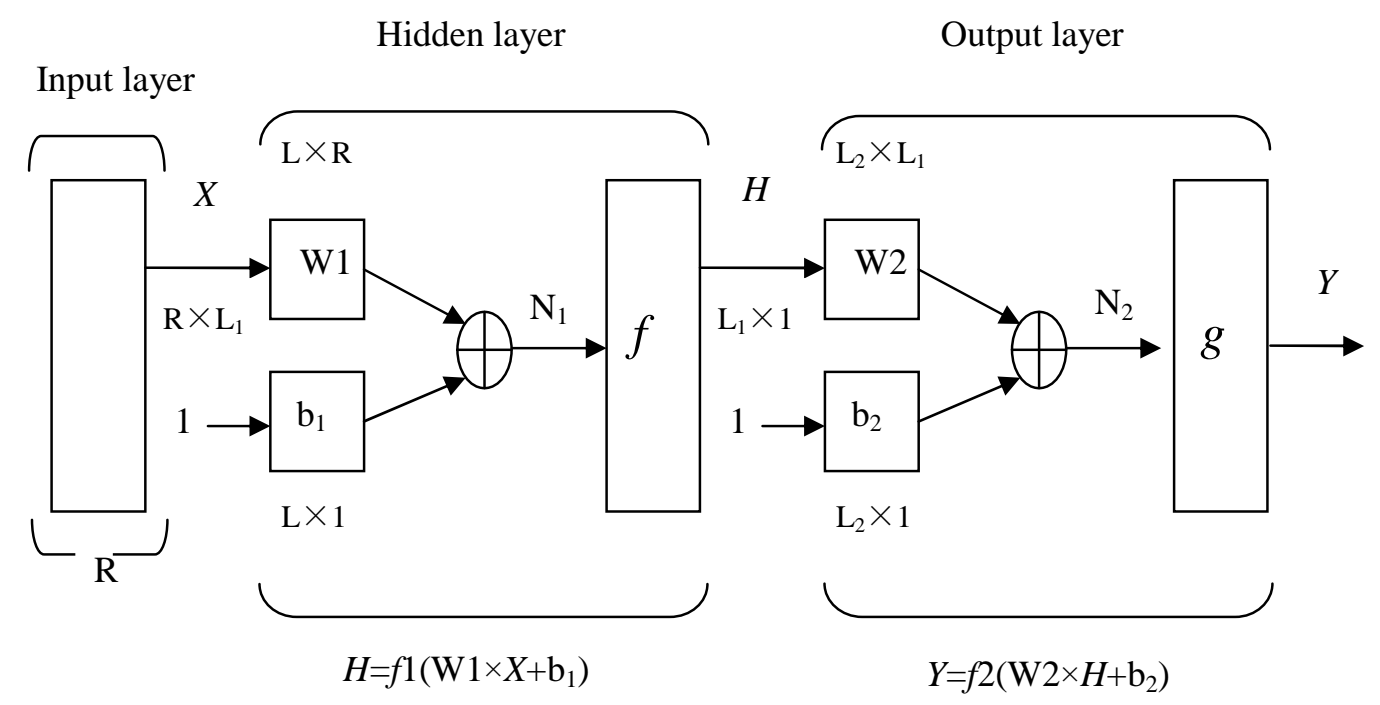

Fig. 1. Structure of multi-layer network with single hidden layer

In this work, concentration and supersaturation were defined as the input vectors, and INT defined as the output vector. The tansig function was used as the hidden propagation function, and 
the purelin function was used as the output propagation function, enabling this ANN model to approximate nonlinear function well.

ANN training was conducted using software MATLAB R2009b developed by MathWorks. In training process of ANN, the values of weights in the model were initialized randomly for the purpose for generating initial model. All weights and biases in the network are obtained through training procedure that was based on the experimentally determined data. When the training error reached the specified level, the training procedure was terminated, and the trained multiple-layer network was then ready to be used for prediction.

To validate the prediction ability of the established ANN model, mean relative error (MRE) defined as follow was used:

$$
M R E=\frac{1}{N} \sum_{1}^{N}\left|\frac{\text { prediction value-experimental value }}{\text { experimental value }}\right|
$$

\section{Results and discussion}

The reliability of prediction results of ANN model is dependent on the number of processing elements in its hidden layers. Therefore, it is necessary to select appropriate number of processing elements. However, it is impossible to determine the number of processing elements directly ${ }^{11}$. The general way to select a suitable number of processing elements is trial. The comparison of MRE of several simulation results indicated that the ANN model with 12 neurons at the hidden layer and 1 neuron in the output layer could give most highly satisfactory result.

As the number of layers and number of units in each layer have been determined, the weights and biases of ANN could be adopted so as to improve the prediction performance of the generated ANN model. The ANN network was trained with limited data obtained from literature. The training results were shown in Tables 1 and 2, and prediction results from the trained network were then validated against test samples.

Table 1 Weights and bias of the hidden layer

\begin{tabular}{cccc}
\hline $\begin{array}{c}\text { Neuron } \\
\text { unit }\end{array}$ & \multicolumn{2}{c}{$\mathrm{W} 1$} & $\mathrm{~b} 1$ \\
\cline { 1 - 3 }$j$ & $\mathrm{~W}_{1 j}$ & $\mathrm{~W}_{2 j}$ & \\
\hline 1 & -3.0989 & 3.6896 & 4.8892 \\
2 & 1.8151 & 4.5078 & -4.0310 \\
3 & -2.7430 & -4.1343 & 2.8871 \\
4 & -2.2804 & -4.1736 & 2.3145 \\
5 & -4.7031 & 0.9355 & 1.5470 \\
6 & 3.2721 & 3.3900 & 0.3088 \\
7 & -1.9698 & 4.3957 & -0.0961 \\
8 & -4.7257 & 1.1386 & -1.3142 \\
9 & 3.4575 & -3.4183 & 2.1919 \\
10 & -4.3738 & 2.2893 & -2.8922 \\
11 & 3.6288 & 3.2143 & 3.9726 \\
12 & -3.3745 & 3.5254 & -4.7759 \\
\hline
\end{tabular}


Table 2 Weights and bias of the output layer

\begin{tabular}{ccc}
\hline Neuron unit & W2 & b2 \\
\cline { 1 - 2 }$j$ & $w_{1 j}$ & \\
\hline 1 & -0.1508 & \\
3 & -0.2162 & \\
4 & 0.0783 & \\
5 & -0.0307 & \\
6 & 0.1672 & \\
7 & -0.3761 & -25.9052 \\
8 & 0.0162 & \\
9 & 0.1878 & \\
10 & 0.1904 & \\
11 & 0.0029 & \\
12 & -0.0271 & \\
\hline
\end{tabular}

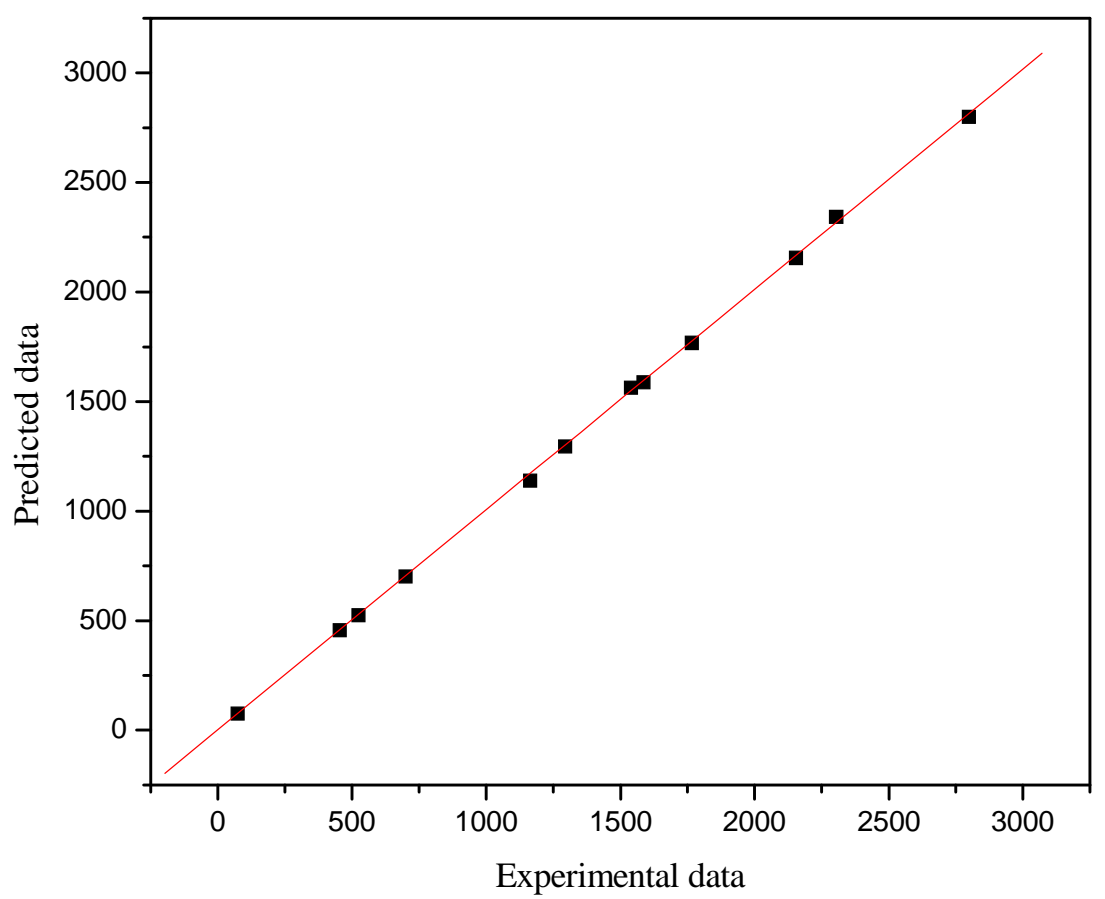

Fig. 2. Training the multilayer ANN network: correlation between the experimental and predicted values of INT.

The model constructed in this case was trained with 15 sets of experimental data. Fig. 2 indicated that the predicted values (the solid line) are in good agreement with the experimentally determined data (the open diamond), and the MRE is only $2.52 \%$. Another 3 sets of experimental data were selected as the test sample to validate the prediction accuracy of the model. The experimental values and predicted values were shown in Fig. 3. All predictions lie within 3.5\% of the experimental values, which indicated that the established ANN model could give good prediction on crystallization induction period. 


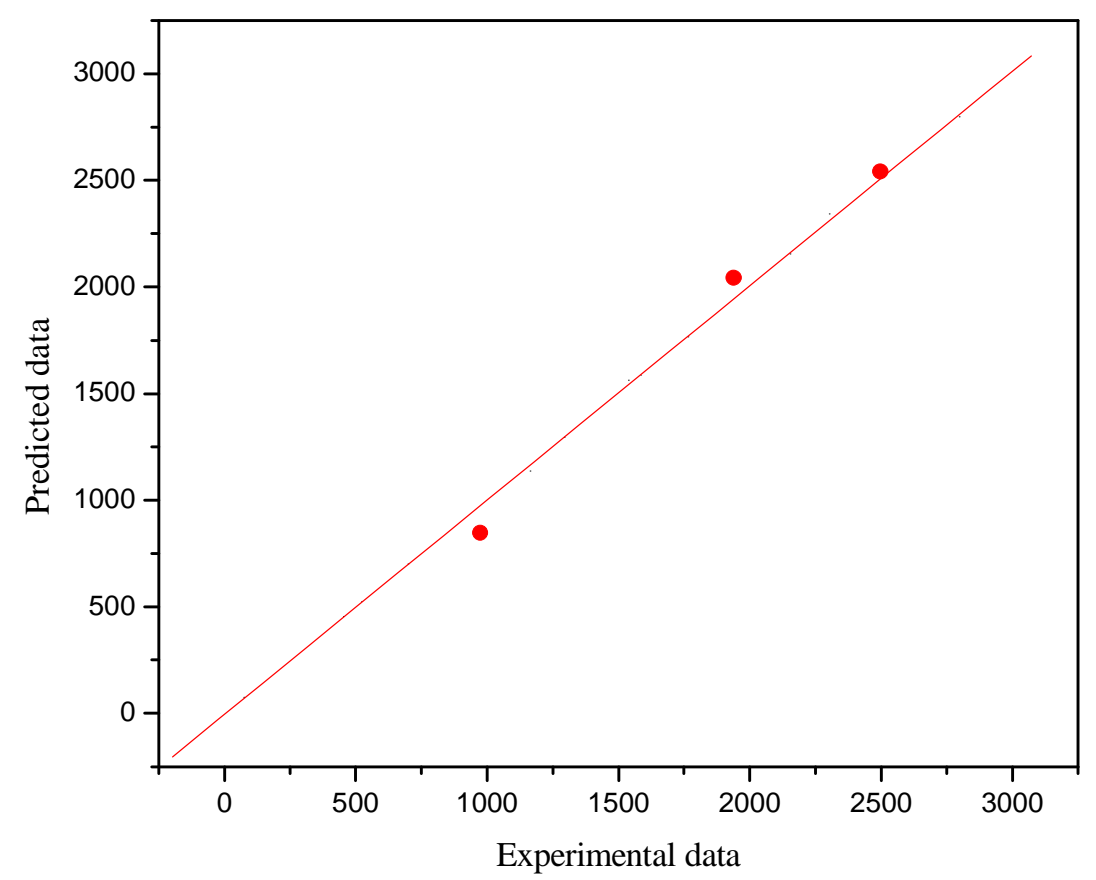

Fig. 3. Multilayer ANN network prediction: comparison between the experimental and predicted values of INT.

\section{Conclusions}

ANN model is able to describe almost any nonlinear system behaviors, which can provide the mapping procedure from input to output. It provides us an effective and applicable way to predict INT of given crystallization condition. An ANN model was developed and trained with limited INT data. The prediction results from the trained ANN model were then validated against another group of measured data. The results confirm that this approach can give accurate predictions of the INT under any specified process condition. Further work will be performed to investigate the applicability of ANN to other process.

\section{Acknowledgements}

This work was financially supported by the Science and Technology Program for Public Wellbeing (Grant No. 2012GS120201).

\section{References}

1. Selvaraju, K.; Valluvan, R.; Kirubavathi, K.; Kumararaman, S., Materials Letters 2007, 61, (14-15), 3041-3044.

2. Lenka, M.; Sarkar, D., Journal of Crystal Growth 2014, 408, 85-90.

3. Chenthamarai, S.; Jayaraman, D.; Ushasree, P. M.; Meera, K.; Subramanian, C.; Ramasamy, P., Materials Chemistry and Physics 2000, 64, (3), 179-183.

4. Selvaraju, K.; Valluvan, R.; Kumararaman, S., Materials Letters 2006, 60, (13-14), 1565-1569.

5. Rajendran, K. V.; Rajasekaran, R.; Jayaraman, D.; Jayavel, R.; Ramasamy, P., Materials Chemistry and Physics 2003, 81, (1), 50-55.

6. Barber, D.; Heskes, T., An introduction to neural networks. 2004,.35-40.

7. Sakas, D. E.; Panourias, I. G.; Simpson, B. A., An introduction to neural networks surgery, a field of neuromodulation which is based on advances in neural networks science and digitised brain 
imaging. In Operative Neuromodulation, Springer: 2007; pp 3--13.

8. Kozma, R.; Bressler, S.; Perlovsky, L.; Venayagamoorthy, G. K., Neural Netw 2009, 22, (5-6), 489-90.

9. Barradas Filho, A. O.; Barros, A. K. D.; Labidi, S.; Viegas, I. M. A.; Marques, D. B.; Romariz, A. R. S.; de Sousa, R. M.; Marques, A. L. B.; Marques, E. P., Fuel 2015, 145, 127-135.

10. Fasel, B., Acta Neurol Belg 2003, 103, (1), 6-12.

11. Murata, N.; Yoshizawa, S.; Amari, S., IEEE Trans Neural Netw 1994, 5, (6), 865-72. 